\title{
Evaluation and exploitation of CryoSat ocean products for oceanographic studies
}

Chris Banks, Francisco Mir Calafat,

Christine Gommenginger, Nadim Dayoub (NOC, UK),

Helen Snaith (BODC/NOC, UK), Andrew Shaw (SKYMAT Ltd. UK),

Paolo Cipollini (Telespazio VEGA UK for ESA, UK),

Jérôme Bouffard (ESA) and Marco Meloni (Serco for ESA, Italy)

chris.banks@noc.ac.uk 


\section{Aims of this presentation}

\section{- Routine CryoSat ocean products reporting}

- Illustrate some examples of validation activity

\section{$>$ Both routine and focussed}

\begin{tabular}{|c|c|c|}
\hline \multicolumn{3}{|c|}{ Remote Sensing of Environment 191 (2017) 131-144 } \\
\hline & Contents lists available at ScienceDirect & \\
\hline s. & Remote Sensing of Environment & \\
\hline ELSEVIER & journal homepage: www.elsevier.com/locate/rse & \\
\hline \multicolumn{3}{|c|}{ Evaluation of new CryoSat-2 products over the ocean } \\
\hline F.M. Calafat ${ }^{\mathrm{a}, *}$ & Bouffard ${ }^{b, c}, H$. Snaith ${ }^{\text {add }}$, P. Féménias ${ }^{b}$ & \\
\hline
\end{tabular}

Calafat et al., 2017

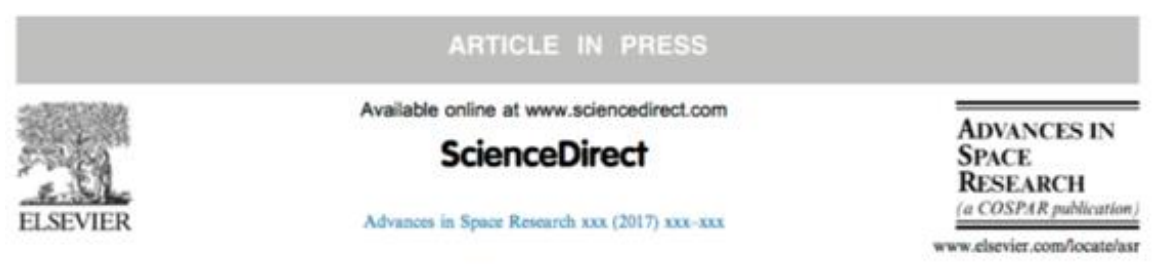

CryoSat ocean product quality status and future evolution

Jerome Bouffard ${ }^{\mathrm{a}, *}$, Marc Naeije ${ }^{\mathrm{b}}$, Christopher J. Banks ${ }^{\mathrm{d}}$, Francisco M. Calafat ${ }^{\mathrm{d}}$, Paolo Cipollini ${ }^{c}$, Helen M. Snaith ${ }^{c}$, Erica Webb ${ }^{f}$, Amanda Hall ${ }^{f}$, Rubinder Mannan ${ }^{f}$, Pierre Féménias ${ }^{8}$, Tommaso Parrinello ${ }^{8}$

Bouffard et al., 2018 


\section{Daily and monthly reports available}

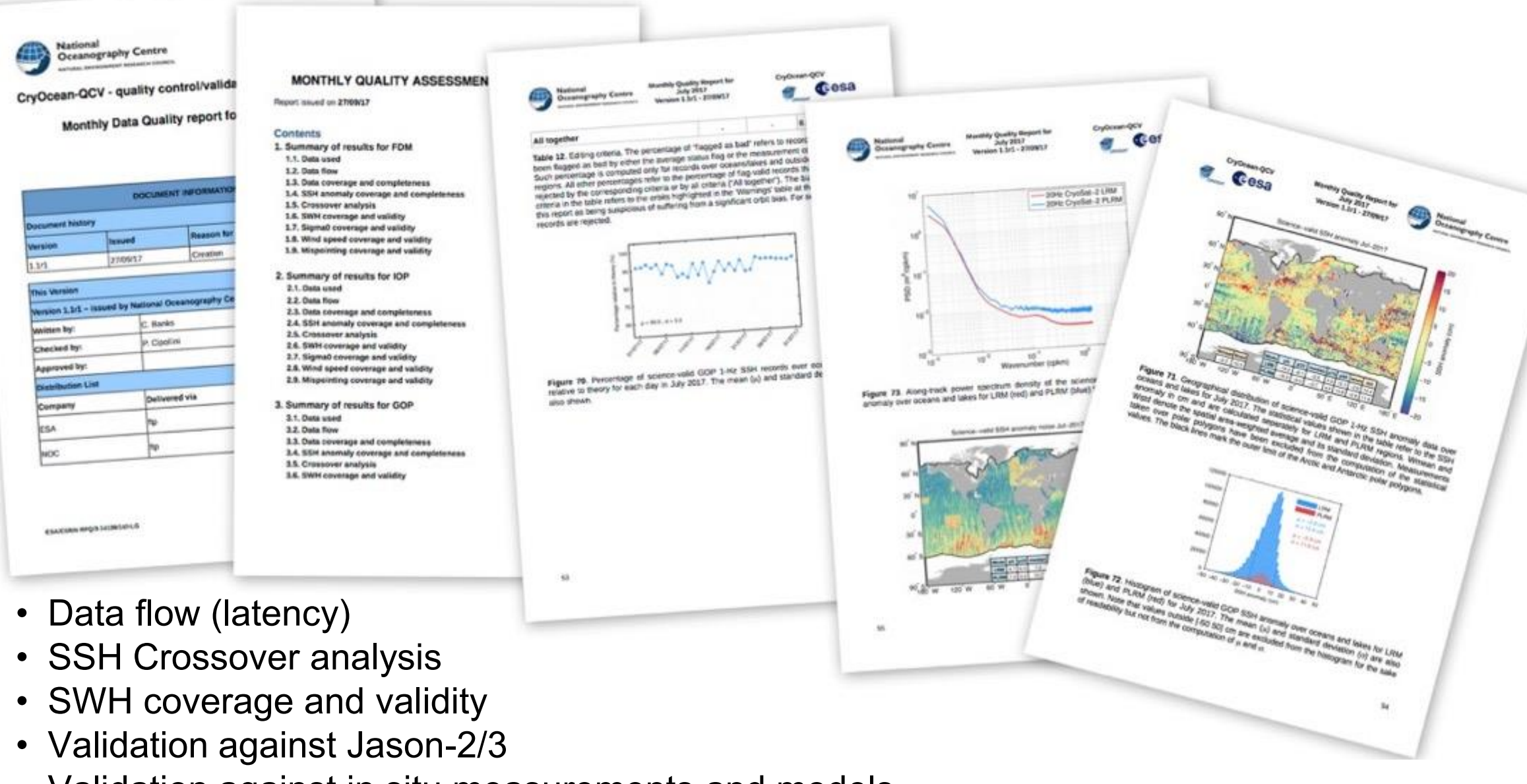

- Validation against in situ measurements and models

tide gauges, wind speed against buoy data, WaveWatch III model data, steric heights derived from T/S Argo profiles 


\section{Noise of altimetric SLA increases with sea state}
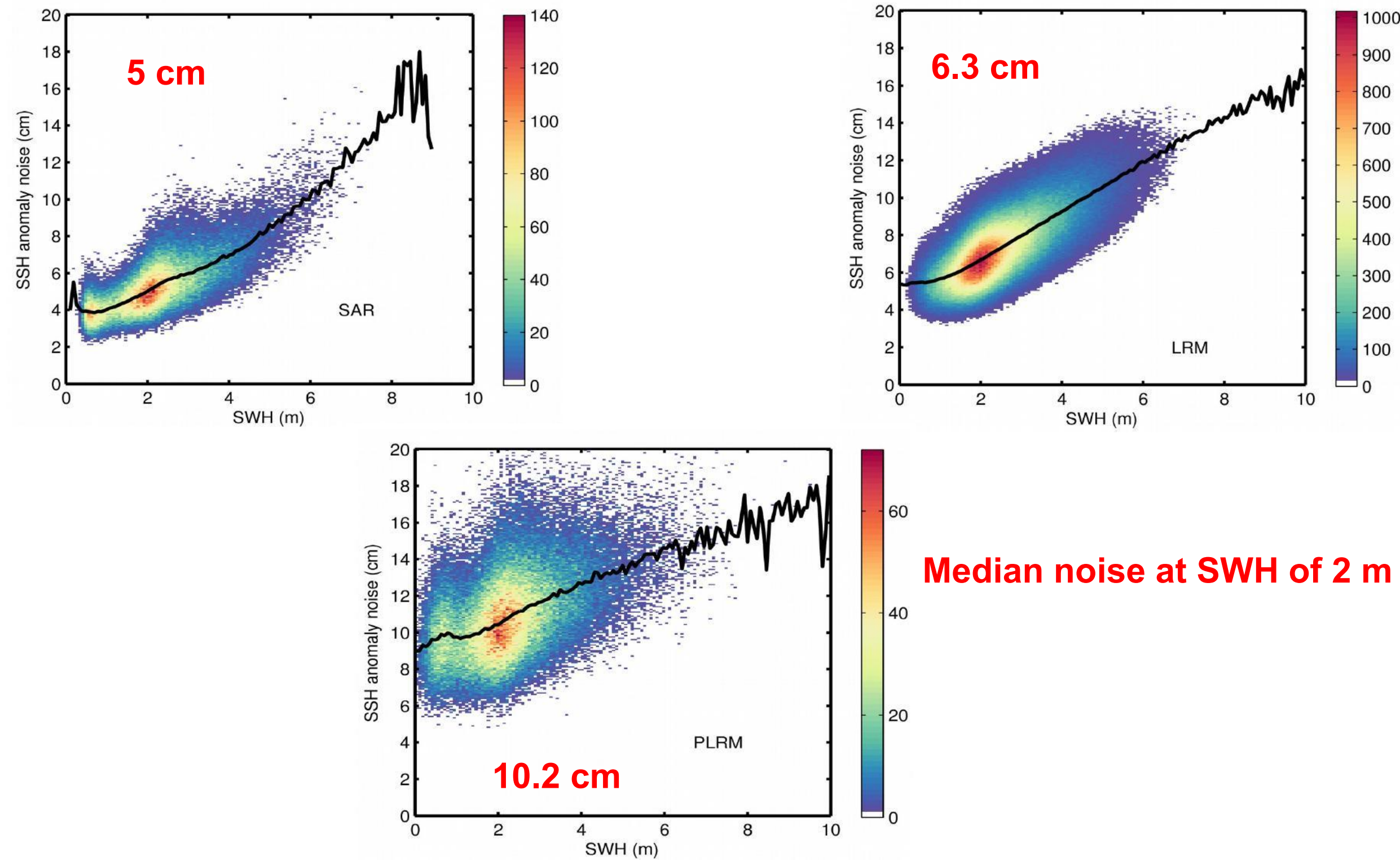

Median noise at SWH of $2 \mathrm{~m}$ 40

20 
Measuring global ocean winds with CryoSat January 2019

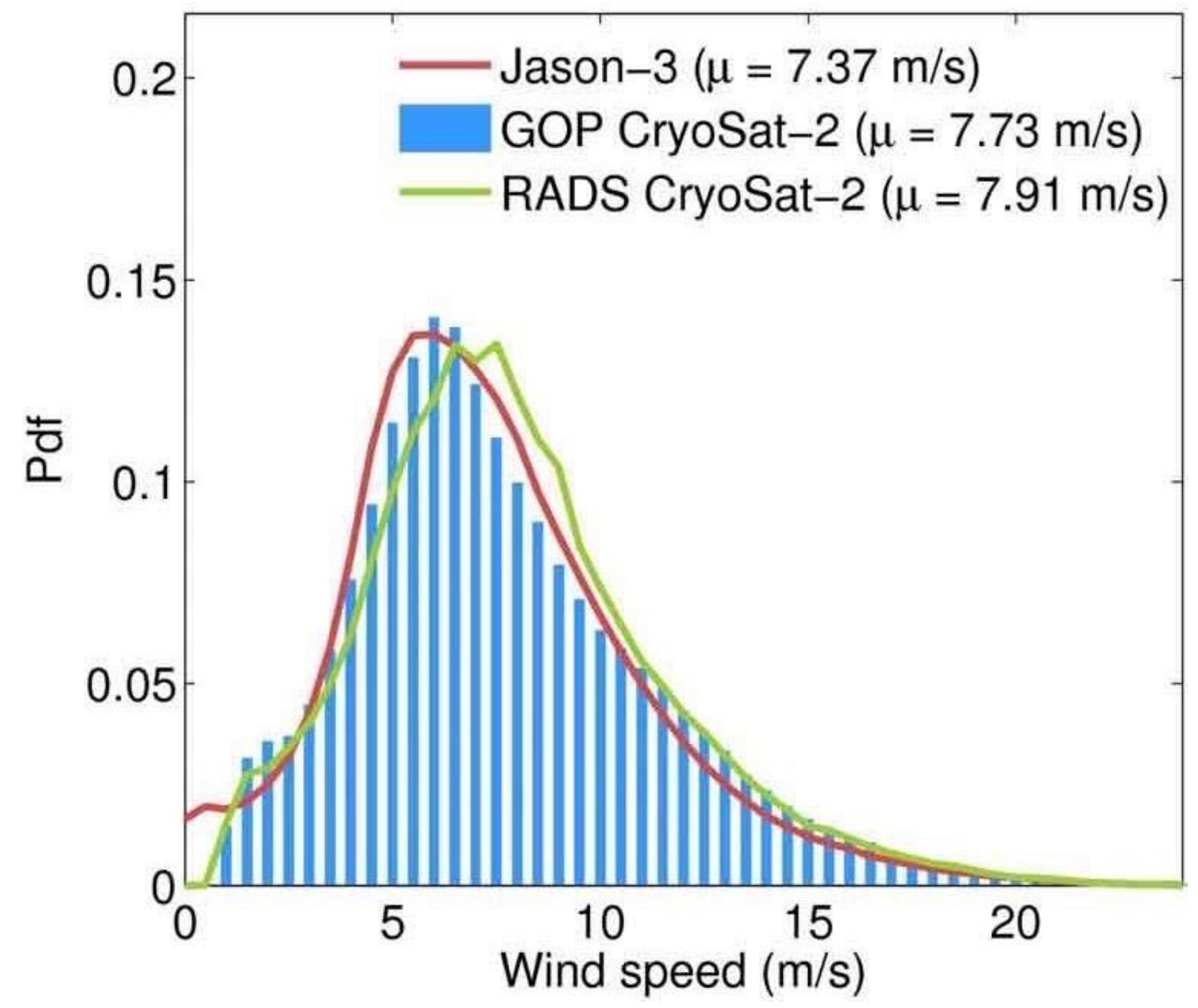




\section{Signal of devastating tsunami observed by CryoSat}

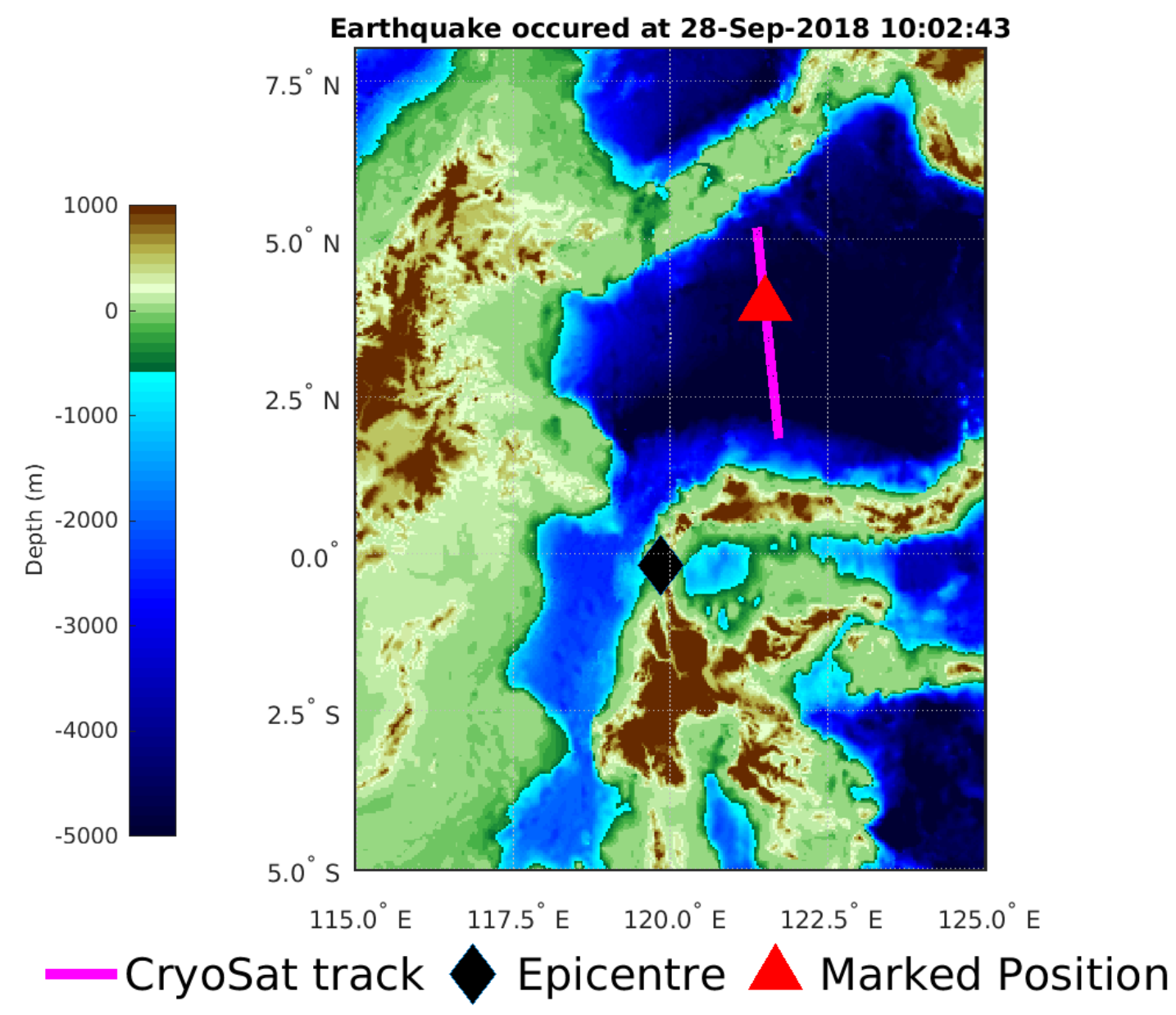

Marked point is 2.4 hours after earthquake and $495.3 \mathrm{~km}$ away
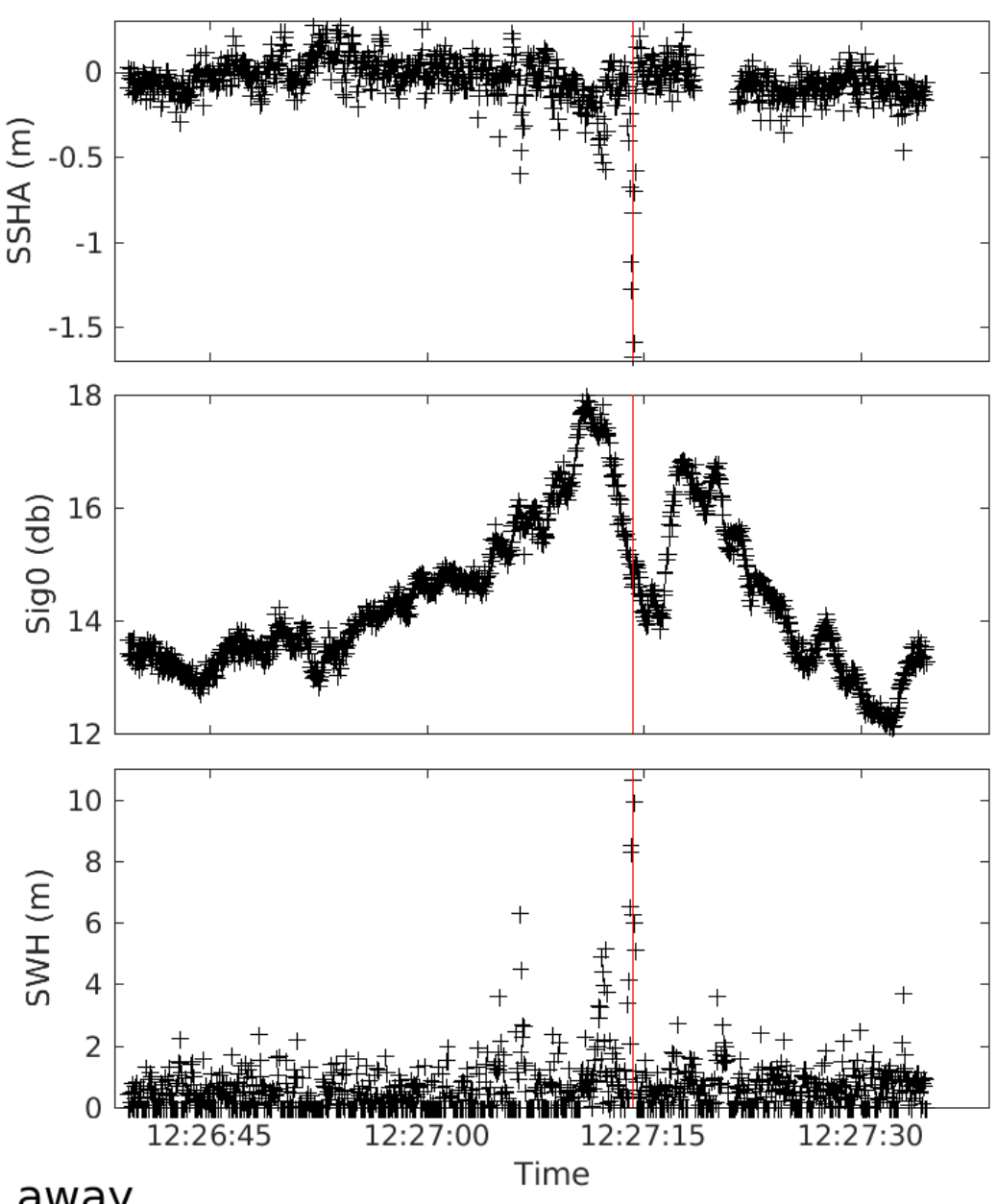

National
Oceanography
Centre 


\section{Rossby Wave Study $\left(65^{\circ}-100^{\circ} \mathrm{E}\right)$}

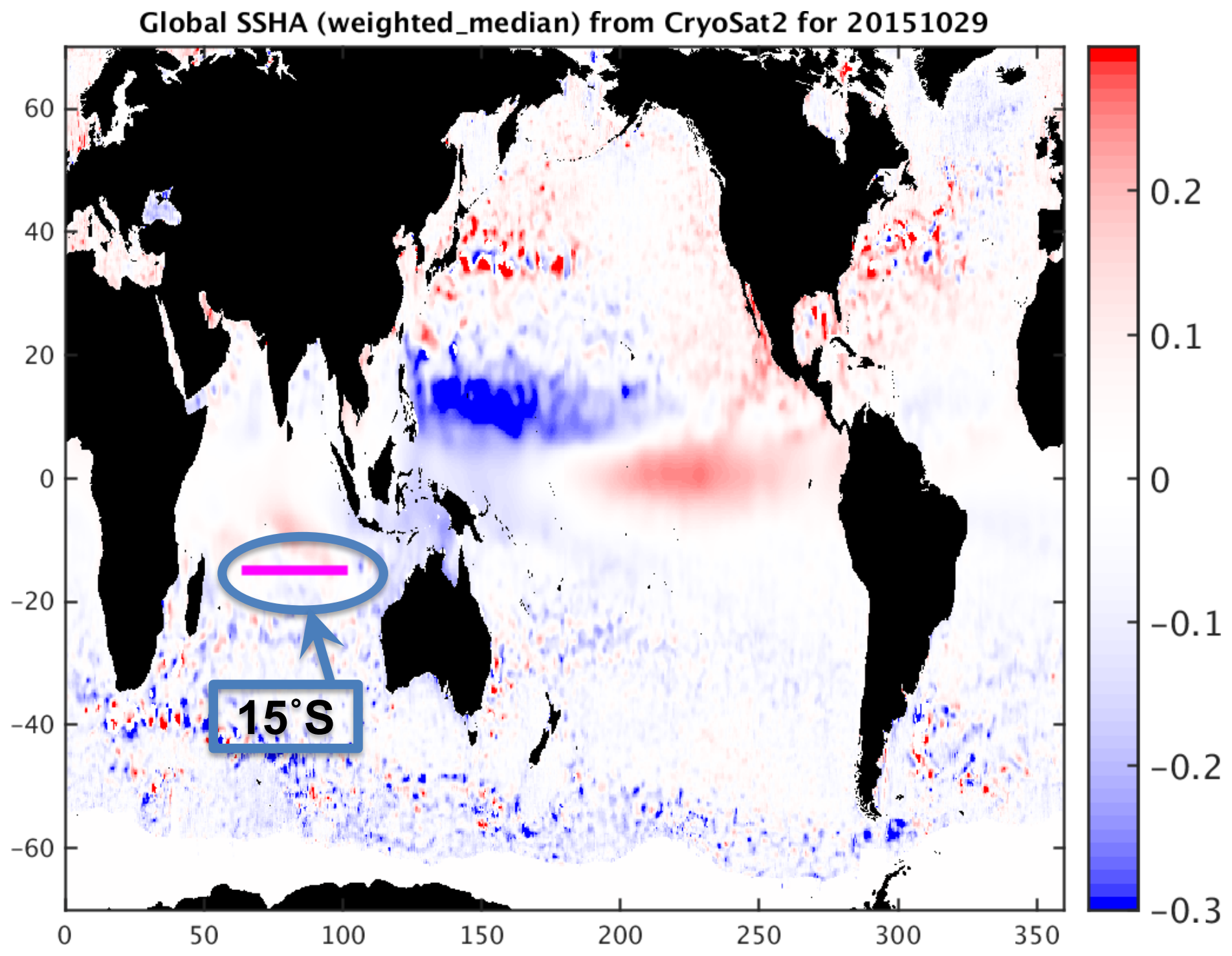




\section{Rossby Wave Study $\left(65^{\circ}-100^{\circ} \mathrm{E}\right)$ $15^{\circ} \mathrm{S}$}

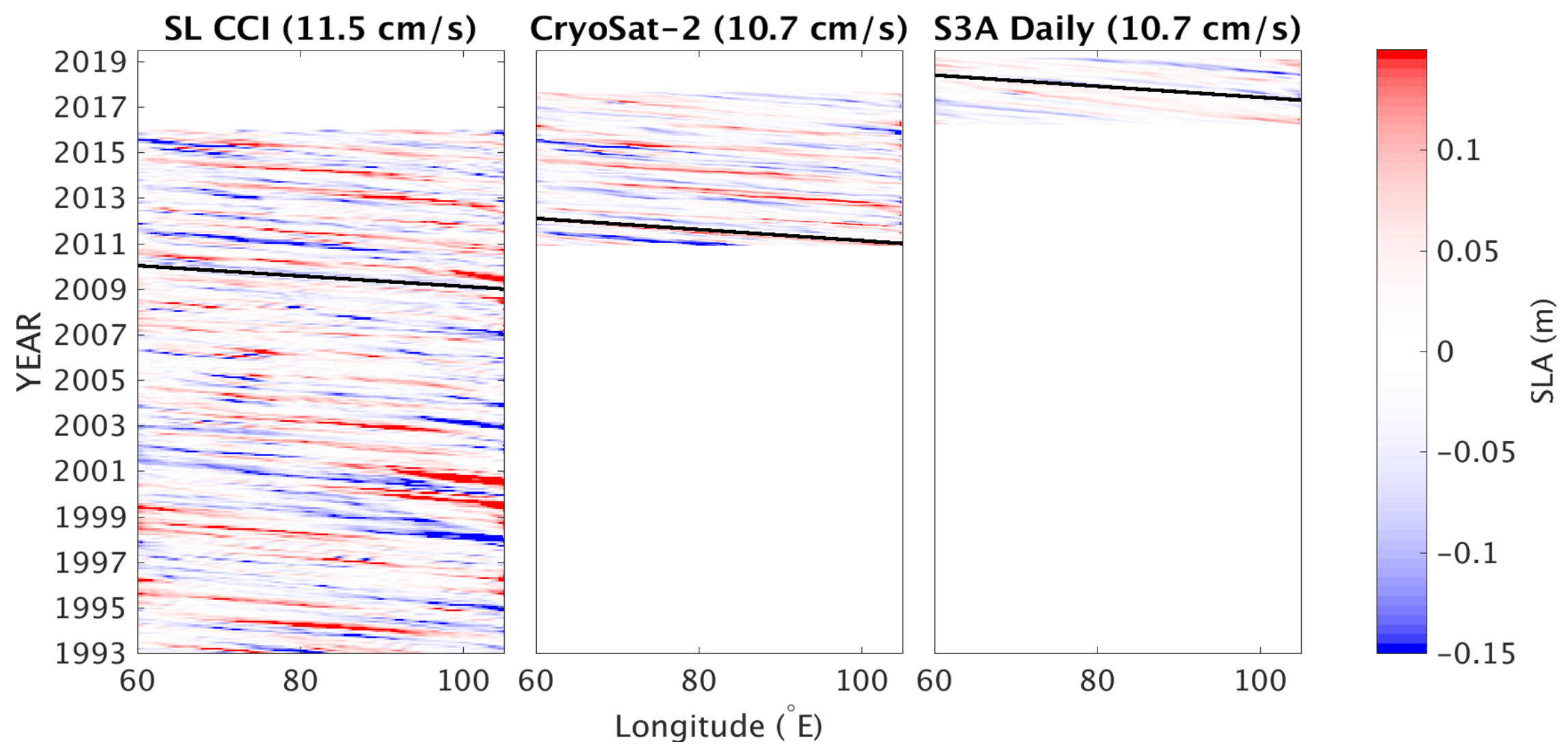




\section{Summary}

- CryoSat Ocean Products available operationally since April 2014 from ESA then reprocessed from start of the mission ( $~ 9$ years of data)

- Excellent performance over ocean

- in terms of noise, compares well with TGs, ARGO, Jason products and other validation sources

- Operational change to Baseline C - including SAR and SARIn full dataset currently being reprocessed

- CryoSat Ocean products ready for oceanographic studies and applications

- CryoSat Ocean Products complement the ocean altimetry record from repeat-orbit missions 